\title{
Magnetic Entropic Forces Emerging in the System of Elemen- tary Magnets Exposed to the Magnetic Field
}

\author{
Edward Bormashenko*
}

${ }^{1}$ Ariel University, Chemical Engineering Department, Engineering Sciences Faculty, Ariel, 407000, Israel, E-mail: edward@ariel.ac.il

\begin{abstract}
Temperature dependent entropic force acting between the straight direct current $I$ and the linear system (string with length of $L$ ) of $N$ elementary non-interacting magnets/spins $\vec{\mu}$ is reported. The system of elementary magnets is supposed to be in the thermal equilibrium with the infinite thermal bath $T$. The entropic force at large distance from the current is given by $F_{m a g n}^{e n}=\frac{N \mu_{0}^{2} \mu^{2} I^{2}}{4 \pi^{2} k_{B} T r^{3}}$, where $r$ is the distance between the edge of the string and the current $I$, and $k_{B}$ is the Boltzmann constant; $(r \gg L$ is adopted). The entropic magnetic force is the repulsion force. The entropic magnetic force scales as $F_{m a g n}^{e n} \sim \frac{1}{T}$, which is unusual for entropic forces. The effect of "entropic pressure" is predicted for the situation when the source of the magnetic field is embedded into the continuous media, comprising elementary magnets/spins.
\end{abstract}

Keywords: entropic force; magnetic field; linear system of elementary magnets; ordering; temperature; repulsion force.

\section{Introduction}

So-called entropic forces attracted attention of investigators in last decades. Entropic force acting in a system is an emergent phenomenon resulting from the entire system's statistical tendency to increase its entropy [1,2]. Entropic force represents the tendency of a system to evolve into a more probable state, rather than simply into one of lower potential energy [1, 2]. A classical example of an entropic force is the temperature dependent elasticity of a freely-jointed polymer chain [3, 4]. For an ideal polymer chain, maximizing its entropy means reducing the distance between its two free ends $[3,4]$. Consequently, an entropic elastic force that tends to collapse the chain is exerted by the ideal chain between its two free ends [3,4]. Muscles of mammals are also driven by entropy forces [5]. As it has been shown, elasticity in the giant muscle protein titin arises from entropy in a way very similar to the entropy-driven elasticity of polymer chains [5]. The so-called "hydrophobic effect" represents additional exemplification of the entropy-forces-driven phenomena. The hydrophobic interaction originates from the disruption of hydrogen bonds between molecules of liquid water by the nonpolar solute [6]. By aggregating together, nonpolar molecules reduce the surface area exposed to water and minimize the effect [6]. This reducing of the surface is thermodinamically (entropically) favorable, giving rise to the clustering of small hydrophobic particles [6]. An interest to entropic force was strengthened after the suggestion of E. Verlinde, who hypothesized the entropic nature of gravity [7]. The entropic origin of gravity was discussed in detail in refs. 8-9. It was shown, that classical Newtonian gravity may be interpreted in terms of an entropic force [8-9]. The entropy origin of gravity was criticized in refs. 10-12 and the problem remains open and debatable. Motivated by Verlinde's theory of entropic gravity, a tentative explanation to the Coulomb's law with an entropic force was suggested [13]. We demonstrate the 
temperature dependent magnetic entropic forces emerging when a string of elementary magnets is exerted to the magnetic field, which tends to order the magnets and in turn to diminish the entropy of the system.

\section{Results and discussion}

Consider the linear (1D) string of elementary non-interacting magnets (spins) $\vec{\mu}$ exerted to the magnetic field generated by a straight, infinite, direct current located, as depicted in Figure 1. We assume that there are $N$ separate and distinct sites fixed in a space and aligned, as shown in Figure 1. Attached to each site is an elementary magnet, which can point only up or down, as shown in Figure 1. The total length of the string is $L$, and the linear density of the magnets $\widetilde{N}$, defined according to Eq. 1 is supposed to be constant along the string.

$$
\widetilde{N}=\frac{N}{L}=\text { const }
$$

The suggested 1D string built of elementary magnets/spins $\vec{\mu}$ is embedded into magnetic field $\vec{H}$ generated by the infinite straight current $H(r)=\frac{I}{2 \pi r}$, as shown in Figure 1 , leading to the spin orientation. The potential energy of a single elementary magnet in the magnetic field is given in the SI system of units by Eq. 2:

$$
U(r)=-\mu_{0} \vec{\mu} \cdot \vec{H}(r)
$$

where $\mu_{0}$ is the vacuum permeability. Assume also that the system of spins is in the thermal equilibrium with the surrounding (thermal bath) under the temperature $T$. Let us divide the string of the magnets into "sub-strings" as follows: let $d N_{i}$ be the number of spins in the sub-string $d r_{i}$ numbered " $i$ ", the magnetic field within the string is $H_{i}\left(r_{i}\right)=\frac{I}{2 \pi r_{i}}$ (distance $r_{i}$ is shown in Figure 1). The entropy of the sub-string $S_{i}$ was addressed in detail in refs. 14-15, and within the approximation of the weak magnetic field, i.e. when $\mu_{0} \mu H \ll k_{B} T$ takes place $\left(k_{B}\right.$ is the Boltzmann constant and $T$ is the temperature), the entropy is given by Eq. 3 (consider $d N_{i}=\widetilde{N} d r_{i}$ ):

$$
S_{i}=S_{0 i}-\frac{\mu_{0}^{2} \mu^{2} H_{i}^{2}\left(r_{i}\right) d N_{i}}{2 k_{B} T^{2}}=S_{0 i}-\frac{\widetilde{N} \mu_{0}^{2} \mu^{2} I^{2} d r_{i}}{8 \pi^{2} k_{B} T^{2} r_{i}^{2}}
$$

where $\boldsymbol{d} \boldsymbol{r}_{\boldsymbol{i}}$ is the length of the "ith" sub-string, $d N_{i}$ is the number of spins in the "ith" sub-string $d r_{i}$, and $\boldsymbol{S}_{\mathbf{0} i}=\boldsymbol{k}_{\boldsymbol{B}}\left[\boldsymbol{N}_{\boldsymbol{i}} \ln \boldsymbol{2}-\frac{1}{2} \boldsymbol{l n} \frac{2}{\pi \boldsymbol{N}_{i}}\right]$ is the constant (it is latently adopted that a sub-string contains a "large" number of spins, enabling the statistical approach; for details see refs 14-15, in which the field of validity of Eq. 3 is carefully addressed). The total entropy $S$ of the string of spins exerted to the magnetic field generated by the infinite straight direct current $I$, depicted in Figure 1, is given by Eq. 4:

$$
S=\sum_{i=1}^{n} S_{i}=S_{0}-\int_{r}^{r+L} \frac{\widetilde{N} \mu_{0}^{2} \mu^{2} I^{2}}{8 \pi^{2} k_{B} T^{2} r^{2}} d r,
$$

where $n$ is the total number of the sub-strings, $L$ is the total length of the string and $\boldsymbol{S}_{\mathbf{0}}=\sum_{i=1}^{n} S_{o i}$. Integration in Eq. 4 yields for the large distances, i.e. $r \gg L$ the following expression (consider Eq. 1 and $\widetilde{N}=$ const):

$$
S=S_{0}-\frac{\widetilde{N} L \mu_{0}^{2} \mu^{2} I^{2}}{8 \pi^{2} k_{B} T^{2} r^{2}}=S_{0}-\frac{N \mu_{0}^{2} \mu^{2} I^{2}}{8 \pi^{2} k_{B} T^{2} r^{2}}
$$

The entropic magnetic force $\boldsymbol{F}_{\boldsymbol{m a g n}}^{\boldsymbol{e n}}$ emerging from Eq. 5 is given by Eq. 6 (see refs. 3$4)$ :

$$
F_{m a g n}^{e n}=-T \frac{\partial S}{\partial r}
$$


Substitution of Eq. 5 into Eq. 6 yields eventually:

$$
F_{\text {magn }}^{e n}=\frac{N \mu_{0}^{2} \mu^{2} I^{2}}{4 \pi^{2} k_{B} T r^{3}}
$$

The eventual Eq. 7 deserves the discussion. First of all, it should be emphasized that the derived magnetic force $F_{m a g n}^{e n}=\frac{N \mu_{0}^{2} \mu^{2} I^{2}}{4 \pi^{2} k_{B} T r^{3}}$ is always the repulsion force; this is intuitively a well-expected prediction. Indeed, whatever is the location of string, the magnetic field always decreases the entropy of the entire string, under ordering of the elementary magnets, which is recognized from Eq. 5, which is thermodynamically unfavorable under isothermal conditions. And the repulsion nature of the entropy magnetic force is independent on the direction of the current $I$, as it is immediately seen from Eq. 7. This result supplies an important prediction: consider the direct current embedded into the continuous medium built of the elementary magnets $\vec{\mu}$, as shown in Figure 2. The magnetic entropic force will repulse elementary magnets, whenever they are located. Thus, in the continuous medium comprising elementary magnets/spins, the phenomenon of the "entropic pressure" stimulated by the external source of the magnetic field (current) is predicted. Recall, that the isothermal pressure of an ideal gas is also a pure entropic phenomenon.

In the exotic case of negative absolute temperatures, corresponding to the population-inverted regime, when a spectrum of the system is bounded, the magnetic entropic force becomes the attraction force [16-17]; however, the separate discussion of this perplexed situation is demanded [18-19].

Secondarily, the entropic magnetic force scales as $\boldsymbol{F}_{\boldsymbol{m a g n}}^{\boldsymbol{e n}}=\frac{\text { const }}{\boldsymbol{T}}$ and this is quite surprising for the entropic forces, which usually are growing with temperature [3-4, 7-13]. The entropic elastic force inherent for an ideal polymer chain appears as:

$$
\vec{F}_{\text {polym }}^{\text {en }}=\frac{3 k_{B} T}{N_{k} b^{2}} \vec{R}
$$

where $N_{k}$ is the number of the Kuhn segments in the chain, $b$ is the length of the Kuhn segment and $\vec{R}$ is the end-to end-distance of the chain [4]. It is seen, that this force scales as $F_{\text {polym }}^{e n} \sim T$, and it is opposite to temperature scaling law recognized from Eq. 7. However, in our case this result is quite predictable; consider, that the temperature movement withstands the magnetic ordering of spins, and thus, prevents their magnetic ordering imposed by the external magnetic field; hence the temperature-related influence is expected to diminish the magnetic entropic force. It is also seen from Eq. 8, that the entropic elasticity in polymers scales as $F_{\text {polym }}^{e n} \sim \frac{1}{N_{k}}$, whereas, the magnetic entropic force scales as $\boldsymbol{F}_{\boldsymbol{m a g n}}^{\boldsymbol{e n}} \sim N$, which is also quite intuitively clear: the growth of the number of the spins strengthens the entire entropic magnetic effect.

And last but not least: the magnetic entropy force scales as $\boldsymbol{F}_{\boldsymbol{m a g n}}^{\boldsymbol{e n}}=\frac{\boldsymbol{c o n s t}}{r^{3}}$, and it grows rapidly with the decrease of the distance $r$. The qualitative reasoning for this result is also quite clear: the smaller the distance between the source of the magnetic field and the string of the spins, the larger is the ordering effect, consequently the larger is decrease in the total entropy of the string which is thermodynamically unfavorable.

An accurate expression for the entropic magnetic force $\boldsymbol{F}_{\boldsymbol{m a g n}}^{\boldsymbol{e n}}$, which holds for an entire range of distances $r$ and arises from Eq. 4 and Eq. 6 is easily derived and appears as:

$$
F_{m a g n}^{e n}=\frac{N \mu_{0}^{2} \mu^{2} I^{2}}{8 \pi^{2} k_{B} T} \frac{2 r+L}{r^{2}(r+L)^{2}},
$$

which for $r \gg L$ is reduced to Eq. 7 . 
It should be taken into account that the magnetic entropic force stems from the entropy gradient (see Eq. 6); the effect of ordering itself will not give rise to the discussed entropic effect. Consider a string of elementary magnets embedded into the ideal solenoid; the magnetic field produced by the solenoid, will order the elementary magnets and an entropy will be uniformly decreased within a string; however, the entropic magnetic force in this case will be zero due to the fact that the gradient of entropy along the string is absent.

\section{Conclusions}

We report the entropic force emerging in the linear system of $N$ elementary non-interacting magnets (spins $\overrightarrow{\boldsymbol{\mu}}$ ) exerted to the external magnetic field $\vec{H}$, which tends to order the spins, and consequently to diminish the entropy of entire system. Thermal equilibrium with the thermal bath $T$ is adopted. We considered the string of elementary non-interacting magnets with the length of $L$, exposed to the permanent magnetic field generated by straight infinite current $I$. The calculation of the magnetic entropic force, arising from the entropy gradient, yielded the expression: $F_{\text {magn }}^{\text {en }}=\frac{N \mu_{0}^{2} \mu^{2} I^{2}}{4 \pi^{2} k_{B} T r^{3}}$, where $r$ is the distance between the current $I$ and the edge of the string of elementary magnets (spins), which holds for the "long distance" approximation, namely when $\boldsymbol{r} \gg \boldsymbol{L}$ is adopted. The entropic magnetic force is the repulsion force. Somewhat surprisingly it scales as $\boldsymbol{F}_{\boldsymbol{m a g n}}^{\boldsymbol{e n}}=\frac{\text { const }}{\boldsymbol{T}}$; consider that the entropic forces usually grow with temperature. This prediction becomes understandable if we take into account that the temperature inspired chaos withstands the magnetic ordering of spins, and, thus, prevents their magnetic ordering imposed by the external magnetic field; hence the increase in temperature is expected to diminish the magnetic entropic force. The magnetic entropic force scales as $\boldsymbol{F}_{\boldsymbol{m a g n}}^{\boldsymbol{e n}} \sim N$, which is also quite intuitively clear: the growth of the number of the spins strengthens the entire entropic magnetic effect. The effect of "entropic pressure" is predicted for the situation when the current is embedded into the continuous media, comprising elementary magnets/spins. The exact expression for the entropic magnetic forces, which is valid for the entire range of distances is supplied.

Author Contributions: “Conceptualization, E.B.; investigation, E. B.; .writing-original draft preparation, E. B. All authors have read and agreed to the published version of the manuscript."

Funding: Please add: "This research received no external funding".

Data Availability Statement: "Not applicable".

Acknowledgments: Author is thankful to Yelena Bormashenko for inspiring discussions and help in preparing the figures.

Conflicts of Interest: “The authors declare no conflict of interest." please state "The funders had no role in the design of the study; in the collection, analyses, or interpretation of data; in the writing of the manuscript, or in the decision to publish the results".

\section{References}

1. Taylor Ph. T.; Tabachnik, J. Entropic forces-making the connection between mechanics and thermodynamics in an exactly soluble model. Eur. J. Phys. 2013, 34, 729.

2. Roos, N. Entropic forces in Brownian motion". Am. J. Physics. 2014, 82 (12), 1161-1166.

3. Pohl, R.W. Mechanik, Akustik and Wärmelehere; Springer: Berlin, Germany, 1964.

4. Rubinstein, M.; Colby R. H. Polymer Physics, Oxford Press, NY, USA, 2003.

5. Tskhovrebova, L.; Trinick, J.; Sleep, J.A.; Simmons, R.M. Elasticity and unfolding of single molecules of the giant muscle protein titin. Nature 1997, 387, 308-312.

6. Chandler, D. Interfaces and the driving force of hydrophobic assembly. Nature 2005, 437, 640-647.

7. Verlinde, E. On the origin of gravity and the laws of Newton. J. High. Energy Phys. 2011, 4, 1-27. 
8. Visser, M. Conservative entropic forces. J. High Energ. Phys. 2011, 140, 2011.

9. Lee, J. W. On the Origin of Entropic Gravity and Inertia. Found. Phys. 2012, 42, 1153-1164.

10. Kobakhidze, A. Gravity is not an entropic force. Phys. Rev. D 2011, 83, 021502(R).

11. Gao, Sh. Is Gravity an Entropic Force? Entropy 2011, 13(5), 936-948.

12. Yang, R. Is Gravity Entropic Force? Entropy 2014, 16(8), 4483-4488.

13. Wang, T. Coulomb force as an entropic force. Phys. Rev. D 2010, 81, 104045.

14. Kittel, Ch. Thermal Physics, Ch. 4., J. Wiley \& Sons, N. Y., 1969.

15. Bormashenko, Ed. Entropy, Information, and Symmetry; Ordered Is Symmetrical, II: System of Spins in the Magnetic Field, Entropy 2020, 22(2), 235.

16. Landau, L.D.; Lifshitz, E.M. Statistical Physics, 3rd ed.; Course of Theoretical Physics; Elsevier: Oxford, UK, 2011; Volume 5.

17. Purcell, E.M.; Pound, R.V. A Nuclear Spin System at Negative Temperature. Phys. Rev. 1951, 81, $279-280$.

18. Dunkel, J.; Hilbert, S. Consistent thermostatistics forbids negative absolute temperatures. Nat. Phys. $2013,10,67-72$.

19. Bormashenko, Ed. What Is Temperature? Modern Outlook on the Concept of Temperature, Entropy 2020, $22(12), 1366$. 
$r$

Figure 1. The linear system (string) of elementary magnets shown with arrows exerted to the permanent magnetic field generated by the infinite direct current $I$ is depicted. Current $I$ is perpendicular to the plane of the drawing. The length of the string is $L$; the distance between the current and the edge of the string is $r$.

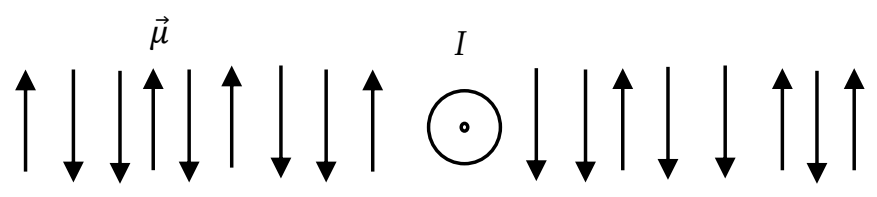


Figure 2. Direct current $I$ embedded into the medium built of magnetic moments $\vec{\mu}$. The magnetic entropic force given by Eq. 9 will repulse the magnetic moments, thus giving rise to the "entropic pressure phenomenon". 